University of Wollongong

Research Online

Faculty of Engineering - Papers (Archive)

Faculty of Engineering and Information

Sciences

$1-1-2008$

\title{
Orientation dependence of the optical spectra in graphene at high
} frequencies

\section{Chao Zhang}

University of Wollongong, czhang@uow.edu.au

Lei Chen

Peking University

Zhongshui Ma

Peking University, Beijing, China, zma@uow.edu.au

Follow this and additional works at: https://ro.uow.edu.au/engpapers

Part of the Engineering Commons

https://ro.uow.edu.au/engpapers/3068

\section{Recommended Citation}

Zhang, Chao; Chen, Lei; and Ma, Zhongshui: Orientation dependence of the optical spectra in graphene at high frequencies 2008, 241402-1-241402-4.

https://ro.uow.edu.au/engpapers/3068

Research Online is the open access institutional repository for the University of Wollongong. For further information contact the UOW Library: research-pubs@uow.edu.au 


\title{
Orientation dependence of the optical spectra in graphene at high frequencies
}

\author{
Chao Zhang* \\ School of Engineering Physics, University of Wollongong, Wollongong, New South Wales 2522, Australia
}

Lei Chen

School of Physics, Peking University, Beijing 100871, China

Zhongshui $\mathrm{Ma}^{\dagger}$

School of Physics, Peking University, Beijing 100871, China

and School of Engineering Physics, University of Wollongong, Wollongong, New South Wales 2522, Australia

(Received 24 September 2007; revised manuscript received 14 May 2008; published 16 June 2008)

\begin{abstract}
On the basis of the Kubo formula we evaluated the optical conductivity of a graphene sheet. The full behavior of frequency as well as temperature dependence of the optical conductivity is presented. We show that the anisotropy of conductivity can be significantly enhanced at high frequencies. The photon absorption depends on the field polarization direction. At the frequency comparable to the maximum separation of upper and lower bands the photon-induced conduction of electrons is strongly suppressed if the polarization of field is along the zigzag direction. The corresponding optical conductivity is several orders of magnitude weaker than that when the light is polarizing along the armchair direction. We propose that the property of orientation selection of absorption in the graphene can be used as a basis for a high-frequency partial polarizer.
\end{abstract}

DOI: 10.1103/PhysRevB.77.241402

PACS number(s): 73.50.Mx, 78.66.-w, 81.05.Uw

Because it is recognized as a new class of materials ${ }^{1}$ in the carbon family, graphene has recently attracted intensive interests as it possesses remarkable electronic properties, such as the anomalous integer quantum Hall effect, ${ }^{2,3}$ the finite conductivity at zero charge-carrier concentration, ${ }^{2}$ and the strong suppression of weak localization, ${ }^{4-7}$ etc. These properties promise building blocks for the technological applications in molecular electronic and optoelectronic devices. In graphene, the conduction and valance bands touch upon each other at isolated points in the Brillouin zone $\left(K\right.$ and $\left.K^{\prime}\right)$. Undoped graphene is a gapless semiconductor, or a semimetal, with vanishing density of states at the Fermi level. Low-energy electronic states of graphene with a linear dispersion at the corner of Brillouin zone are described by the "relativistic" massless Dirac equation. This relativistic kinematical description of graphene is confirmed in quantum Hall studies ${ }^{8}$ and gives us theoretical insight into exotic transport, ${ }^{9}$ magnetic correlation, ${ }^{10,11}$ and dielectric ${ }^{12}$ properties observed in this material. On the theoretical side, most of the works in studies of graphene is based on "massless Dirac theory" in the vicinity of the $K$ point in the Brillouin zone, where all physical quantities remain isotropic. The purpose of this Rapid Communication is to reveal transport properties beyond the regime of linear energy dispersion. Because the carrier concentration in graphene can be varied over a wide range by doping and by the electric field effect, ${ }^{1}$ this makes high-energy excitations in graphene important and interesting. We shall show that the current response is highly anisotropic away from the $K$ point and the high-frequency spectra can reveal a variety of rich physics and anomalous phenomena that electrons and holes possess in graphene.

Besides its transport properties, the gapless energy spectrum of electrons and holes in graphene can lead to specific features of optical and plasma properties. ${ }^{13,14}$ Very recently, it has been demonstrated experimentally that both the optical conductance ${ }^{15}$ and the transmission coefficient ${ }^{16}$ of graphene in the frequency range of $700-400 \mathrm{~nm}$ are very close to constant values. It is anticipated that the optical spectrum induced by elementary electronic excitation can be used to determine the electronic properties of graphene. It is known that optical conductivity is one of the central quantities to determine almost all optical properties of an electron and/or hole system. For a case where the optical transition is induced mainly by a dielectric response of the carriers through the carrier-carrier interaction, the optical conductivity can be obtained simply from the Kubo formula in which the currentcurrent correlation is mainly caused by carrier interactions with a weak external light field. ${ }^{17}$

We present here one interesting property of graphene at high frequencies. On the basis of current-current correlation function we investigate the optical spectrum of graphene. We obtained the full frequency and temperature dependence of the optical spectra for graphene with varying Fermi energy. The optical spectrum shows a cusplike maximum at $\hbar \omega_{c}$ $=2 \times\left(2 \hbar v_{F} / \sqrt{3} a\right)$, where $v_{F}$ is the Fermi velocity and $a$ ( $=\sqrt{3} b$ with $b$ the $c-c$ bond length) is the lattice constant. However, the absorption beyond $\omega_{c}$ is negligible for photons polarizing along the zigzag direction. For light polarizing along the armchair direction there is an absorption continuum which jumps abruptly at $3 \hbar \omega_{c}$, corresponding to the vertical transition at $\mathbf{k}=0$. Our results of anisotropic absorption demonstrate that graphene can be used as a partial polarizer in the high-frequency regime where the absorption along the armchair direction is finite while the absorption along the zigzag direction is basically zero. We analyze this conductivity anisotropy in terms of the energy dependence of the interband velocity operator.

A unit cell, whose wave vectors are given by $\mathbf{a}_{1}$ $=a(\sqrt{3} / 2,1 / 2)$ and $\mathbf{a}_{2}=a(\sqrt{3} / 2,-1 / 2)$, in a honeycomb lattice contains two atoms denoted as A and B. In this Rapid Communication the $y$ axis is considered to be along the line connecting atoms of the same sublattice while the $x$ axis is 


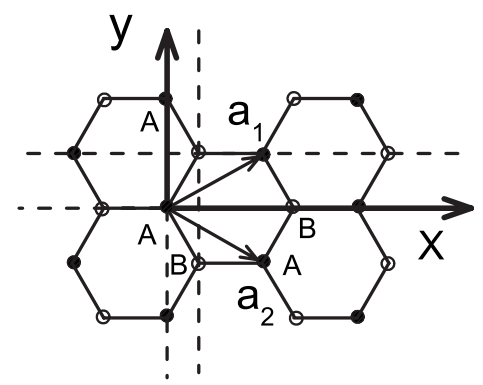

FIG. 1. Polarization direction in graphene, the $x$ axis is the armchair direction and the $y$ axis is the zigzag direction.

along the line connecting atoms of alternating sublattice, as shown in Fig. 1. We assume the graphene is intrinsic, i.e., free of impurity doping. For the case of zero carrier density the two bands cross the Fermi level (setting as zero) at $\mathbf{K}$ and $\mathbf{K}^{\prime}$ points of the first Brillouin zone, whose wave vectors are given by $\mathbf{K}=(4 \pi / 3 a)(1 / \sqrt{3}, 1)$ and $\mathbf{K}^{\prime}$ $=(4 \pi / 3 a)(3 / 2 \sqrt{3},-1 / 2)$. In the presence of applied electric field, the chemical potential can be moved to either positive or negative regime. The Hamiltonian of graphene is given as

$$
H_{0}=\left(\begin{array}{cc}
0 & h(\mathbf{k}) \\
h^{*}(\mathbf{k}) & 0
\end{array}\right),
$$

with $h(\mathbf{k})=-t\left[1+e^{i \mathbf{k} \cdot \mathbf{a}_{1}}+e^{i \mathbf{k} \cdot \mathbf{a}_{2}}\right]$. The eigenfunctions and the eigenvalues can be written as $\xi_{\mathbf{k}, s}=(1 / \sqrt{2})\left(-s(t /|t|) e^{i \varphi_{\mathbf{k}}}, 1\right)^{T}$ and $\quad \epsilon_{\mathbf{k}, s}=s \epsilon_{0} \sqrt{3+\alpha(\mathbf{k})}, \quad$ where $\quad s= \pm 1, \quad \tan \varphi$ $=\tan ^{-1}\left(\sin \mathbf{k} \cdot \mathbf{a}_{1}+\sin \mathbf{k} \cdot \mathbf{a}_{2}\right) /\left(1+\cos \mathbf{k} \cdot \mathbf{a}_{1}+\cos \mathbf{k} \cdot \mathbf{a}_{2}\right), \quad \alpha(\mathbf{k})$ $=-2+4 \cos \sqrt{3} k_{x} a / 2 \cos k_{y} a / 2+4 \cos ^{2} k_{y} a / 2$, and $\epsilon_{0}=|t| . v_{F}$ is the Fermi velocity, which relates to the hopping parameter, i.e., $v_{F}=(\sqrt{3} a / 2 \hbar)|t|$.

In the presence of a uniform time-dependent electric field $\mathbf{E}=\mathbf{E}_{0} e^{-i \omega t}$, the second quantized Hamiltonian is obtained $H=\sum_{\mathbf{k}, s} \epsilon_{\mathbf{k}, s} a_{\mathbf{k}, s}^{\dagger} a_{\mathbf{k}, s}+(1 / c) \mathbf{A} \cdot \mathbf{J}$, where $\mathbf{A}=(c / i \omega) \mathbf{E}$. The components of the current density operator $\mathbf{J}$ can be written as

$$
\begin{aligned}
J_{x}= & -2 e v_{F} \sum_{\mathbf{k}, s} s \frac{\sin \frac{\sqrt{3}}{2} k_{x} a \cos \frac{1}{2} k_{y} a}{\sqrt{3+\alpha(\mathbf{k})}} a_{\mathbf{k}, s}^{\dagger} a_{\mathbf{k}, s} \\
& +i 2 e v_{F} \sum_{\mathbf{k}, s} s \frac{1+\cos \frac{\sqrt{3}}{2} k_{x} a \cos \frac{1}{2} k_{y} a+\cos k_{y} a}{\sqrt{3+\alpha(\mathbf{k})}} a_{\mathbf{k}, s}^{\dagger} a_{\mathbf{k},-s}
\end{aligned}
$$

and

$$
\begin{aligned}
J_{y}= & -\frac{2 e v_{F}}{\sqrt{3}} \sum_{\mathbf{k}, s} s \frac{\cos \frac{\sqrt{3}}{2} k_{x} a \sin \frac{1}{2} k_{y} a+\sin k_{y} a}{\sqrt{3+\alpha(\mathbf{k})}} a_{\mathbf{k}, s}^{\dagger} a_{\mathbf{k}, s} \\
& -i \frac{2 e v_{F}}{\sqrt{3}} \sum_{\mathbf{k}, s} s \frac{\sin \frac{\sqrt{3}}{2} k_{x} a \sin \frac{1}{2} k_{y} a}{\sqrt{3+\alpha(\mathbf{k})}} a_{\mathbf{k}, s}^{\dagger} a_{\mathbf{k},-s} .
\end{aligned}
$$

From the above expression it is seen that for a graphene under an optical field, two kinds of transitions (intraband and interband) contribute to the current response. The Kubo formula for the dynamic conductivity is given as

$$
\sigma_{\mu \nu}(\omega)=(1 / \omega) \int_{0}^{\infty} d t e^{i \omega t}\left\langle\left[J_{\mu}(t), J_{\nu}(0)\right]\right\rangle .
$$

Based on the current operators given in Eqs. (2) and (3), it is found that the intraband terms do not contribute to the conductivity in graphene free of any disorders. The electron conduction is solely due to the electron jumping between the bands with the absorption of a photon. After some algebra, we found

$$
\begin{aligned}
\sigma_{x x}= & \frac{e^{2} v_{F}^{2}}{\pi^{2} \omega} \int d \mathbf{k} \frac{\left(1+\cos \frac{\sqrt{3}}{2} k_{x} a \cos \frac{1}{2} k_{y} a+\cos k_{y} a\right)^{2}}{3+\alpha(\mathbf{k})} \\
& \times\left(\frac{f_{\mathbf{k},+}-f_{\mathbf{k},-}}{\hbar \omega+2 \epsilon_{0} \sqrt{3+\alpha(\mathbf{k})}}-\frac{f_{\mathbf{k},+}-f_{\mathbf{k},-}}{\hbar \omega-2 \epsilon_{0} \sqrt{3+\alpha(\mathbf{k})}}\right)
\end{aligned}
$$

and

$$
\begin{aligned}
\sigma_{y y}= & \frac{e^{2} v_{F}^{2}}{3 \pi^{2} \omega} \int d \mathbf{k} \frac{\sin ^{2} \frac{\sqrt{3}}{2} k_{x} a \sin ^{2} \frac{1}{2} k_{y} a}{3+\alpha(\mathbf{k})}\left(f_{\mathbf{k},+}-f_{\mathbf{k},-}\right) \\
& \times\left[\frac{1}{\hbar \omega+2 \epsilon_{0} \sqrt{3+\alpha(\mathbf{k})}}-\frac{1}{\hbar \omega-2 \epsilon_{0} \sqrt{3+\alpha(\mathbf{k})}}\right]
\end{aligned}
$$

with $\omega=\omega+i 0_{+}$, where $f_{\mathbf{k}, s}$ is Dirac-Fermi distribution function. In addition, $\sigma_{x y}=\sigma_{y x}=0$ because of symmetry.

From the forms of dynamic conductivity we identify immediately our first result that the optical absorption for photons polarizing along $x$ direction is quite different from that when photons are polarizing along the $y$ direction. To see this polarization dependence of the conductivity we calculate the real and imaginary parts of dynamic conductivity numerically. With an energy unit $\epsilon_{0}$ the temperature is scaled as $T_{0}=k_{B} T / \epsilon_{0}$. The frequency dependence of the real part of the optical conductivity $\operatorname{Re} \sigma_{x x}(\omega)$ and $\operatorname{Re} \sigma_{y y}(\omega)$ are shown in Fig. 2, with two different chemical potentials. It is found that the photon absorption starts from $\omega=0$ and increases with $\omega$ for a graphene with $\mu=0$ [Figs. 2(a) and 2(c)] at low temperature, which is consistent with the metallic absorption characteristics. However, for a graphene with nonzero $\mu$ [see Figs. 2(b) and 2(d)] there is a threshold frequency $\omega_{\text {th }}$ $=2 \mu / \hbar$ and the absorption is almost negligible when the photon frequency is below $\omega_{t h}$. The reason is that the allowed transitions only involve vertical processes, $\hbar \omega=\epsilon_{\mathbf{k}, s}-\epsilon_{\mathbf{k}, s^{\prime}}$. As temperature increases, the phase space for electronic transitions for $\omega<2 \mu$ increases and finite absorption is observed. Both $\operatorname{Re} \sigma_{x x}(\omega)$ and $\operatorname{Re} \sigma_{y y}(\omega)$ exhibit a sharp absorption maximum at $\omega=2 \epsilon_{0} / \hbar$. This corresponds to vertical transitions at $k_{y} a=\pi$ in the energy contour of the reciprocal lattice. The joint density of state (JDOS) for electron-hole excitations reaches the cusp-like maximum at this energy due to the maximum $k_{x}$ degeneracy at $k_{y} a=\pi$. As a result the absorption is sharply peaked with a cusp shape. When the 

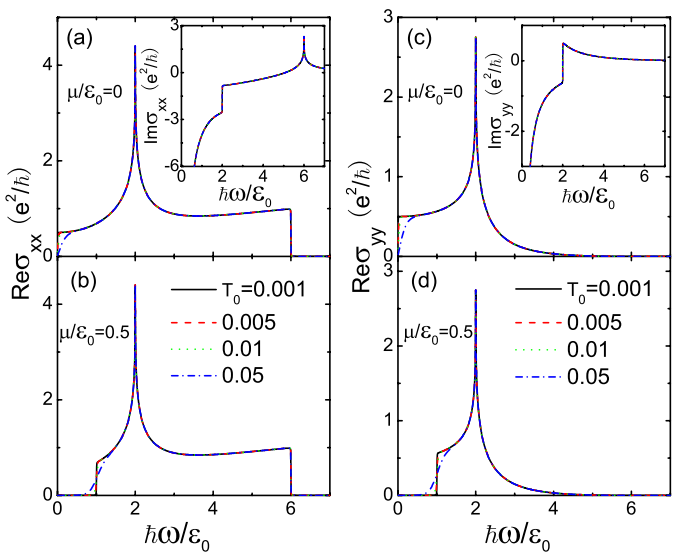

FIG. 2. (Color online) (a) and (c): The real part of the optical conductivity $\sigma_{x x}$ and $\sigma_{y y}$ vs frequency at various temperatures for the Fermi energy lies on the $K$ point. Inset: the imaginary part of the optical conductivity $\sigma_{x x}$ and $\sigma_{y y}$ vs frequency. (b) and (d): Same as (a) and (c) but the Fermi energy lies in the conduction band.

frequency $\omega$ exceeds $2 \epsilon_{0} / \hbar$ it is found that $\operatorname{Re} \sigma_{x x}(\omega)$ and $\operatorname{Re} \sigma_{y y}(\omega)$ have very different frequency dependence. The $\omega$ dependence of $\operatorname{Re} \sigma_{x x}$ increases slowly with $\omega$ at high $\omega$ and jumps abruptly to zero at $\omega=6 \epsilon_{0} / \hbar$, a feature due to the interplay of the increase interband velocity component $v_{x}$ and decreasing DOS. The jumping point corresponds to the maximum energy gap in energy contour. In contrast to the $\operatorname{Re} \sigma_{x x}(\omega)$ there is no jumping in the $\operatorname{Re} \sigma_{y y}(\omega)$ and it tends to vanish rapidly for $\omega>2 \epsilon_{0} / \hbar$. The reason for this striking difference between the $\operatorname{Re} \sigma_{x x}(\omega)$ and the $\operatorname{Re} \sigma_{y y}(\omega)$ at high frequency lies in the energy dependence of the interband velocity operator $\mathbf{v}_{s,-s}$ [referring to the second term of Eqs. (2) and (3)]. For the $x$ component of current $j_{x}$, the corresponding velocity $v_{s,-s}^{x}$ increases as energy increases and reaches its maximum which coincides with the maximum separation of energy between conduction and valance bands at $\mathbf{k}=0$. Differing from $v_{s,-s}^{x}$ the $v_{s,-s}^{y}$ for $j_{y}$ decreases rapidly with energy at high frequency. As a result, although the absorbing $y$ polarized photon at high frequency is energetically possible, the excited electrons are not allowed to gain any velocity along $y$ direction. This polarization-dependent absorption is not only most significant at low to room temperature, but can also survive up to a very high temperature.

The cusplike maximum in the optical spectral is unique to the honeycomb lattice. The derivative at cups is discontinuous. According to the Kramers-Kronig relations, a discontinuous derivative in the real part requires that the imaginary part has steplike behaviors in the imaginary part, and vice versa. This has been confirmed by the inset in Figs. 2(a) and $2(\mathrm{c})$. For a given $\omega$, the spectrum is nonzero within the regime of $-\hbar \omega / 2<\mu<\hbar \omega / 2$. At low temperature, the conductivity takes on a value of order $\pi e^{2} / h$, which can be viewed as a frequency-dependent analog of minimum conductivity. ${ }^{2}$ At fixed frequency, it is found that the optical spectra shows a narrow plateau at low temperature then decreases rapidly with temperature at high temperatures. The number of carrier in the valance band at a given transition energy, $\epsilon$, is proportional to $\exp \left(-\epsilon / k_{B} T\right)$. Therefore, the optical spectra decay exponentially with the increase of temperature.
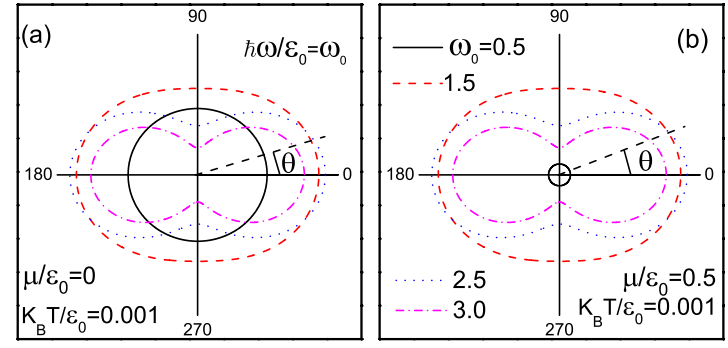

FIG. 3. (Color online) The angular dependence of the absorption coefficient, $\operatorname{Re} \sigma_{l}(\theta)$.

The electromagnetic absorption is given by the real part of the longitudinal conductivity along arbitrary direction. The polarization-dependent absorption strength can be best viewed in the contour diagram shown in Fig. 3. The anisotropy is slightly stronger for $\mu \neq 0$. The angular variation of the absorption leads to a transmitted field whose intensity changes with the polarization direction. The angulardependent intensity of the transmitted field through a graphene is shown in Fig. 4. Within the frequency range of $2|t|<\omega<6|t|$, the higher the frequency is, the stronger the anisotropy in the transmitted field.

Saito et al. ${ }^{18}$ have show that around the $K$ and $K^{\prime}$ points the optical transition matrix elements of graphene depends on the magnitude and direction of the wave vector. Hence one might expect that the total absorption (at given frequency for all $\mathbf{k}$ points) should be anisotropic. However our results indicate that this expectation is incomplete. Contrary to their expectation, we found that although the absorption matrix elements are strongly $\mathbf{k}$ dependent around $K$ and $K^{\prime}$ points, the total absorption at low energy (which is the sum of absorption matrix elements around $K$ and $K^{\prime}$ points) is actually quite isotropic. The anisotropy becomes significant only for frequencies above the hopping bandwidth. Quantitatively, this anisotropy $\left(\operatorname{Re} \sigma_{x x} / \operatorname{Re} \sigma_{y y}\right)$ can reach several orders of magnitude at high frequencies. Very recently Oyama $^{19}$ et al. reported a calculation of absorption of single wall nanotubes. They found that the absorption varies with the chirality and size of the tube. This variation is about a factor of 2 for small tubes and becomes negligible as tube
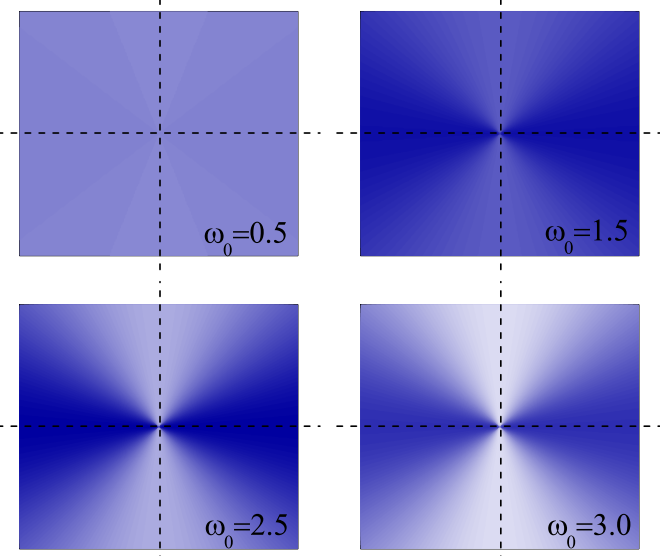

FIG. 4. (Color online) The angular dependence of the transmitted field. The lightness represents the absolute intensity. $\mu / \epsilon_{0}=0.0$ 
diameter increases. In another words, for tubes with a very large diameter (which is an equivalence to graphene), they found no polarization dependence for the absorption. Our result shows the polarization dependence is very strong for an infinitely large tube as long as the frequency is in the range of $2|t|<\omega<6|t|$.

The high-frequency polarization effect of graphene presented here is different than that of commercial polarizer currently in use. Most commercial polarizer has complete polarization effect, i.e., the intensity of a white source will be reduced to half after polarizer and light will be linearly polarized. The effect of graphene is to absorb light polarizing along the armchair direction at the rate of one to two orders magnitude stronger than that along the zigzag direction, (e.g, at $\quad \omega=4|t|, \quad \operatorname{Re} \sigma_{x x} / \operatorname{Re} \sigma_{y y} \approx 25$ and for $\omega=5|t|$, $\operatorname{Re} \sigma_{x x} / \operatorname{Re} \sigma_{y y} \approx 200$ ). So it will only produce a partially polarized light. Recent experiments ${ }^{20,21}$ indicate that one layer of graphene is almost transparent at $\omega$ up to $0.5|t|$. This does not in anyway contradict our result of polarization and absorption at high frequencies. Our result of the absorption rate of armchair polarization by one layer of graphene for $3|t|<\omega<6|t|$ is about $10 \%$. This is significantly higher than that at $\omega=0.5|t|$ and should be measurable. However a single layer of graphene with $10 \%$ maximum absorption along the armchair direction is probably not enough to alter the nearly transparency even at high frequencies. In practice, the best effect can be achieved with the use of multilayers. A system consisting of 5-10 layers should be sufficient in reducing the intensity along the armchair direction by $50 \%$ while keeping the intensity along the zigzag direction unchanged.

In conclusion, we have studied the optical conductivity of graphene and shown its orientation dependence in frequency for photons polarizing along the armchair direction or along the zigzag direction. We show evidently that the absorption vanishes rapidly for frequencies above $2|t|$, in comparison to that along the armchair direction, if the photons are polarized along the zigzag direction. As a consequence, the orientation selection of excited electron transport indicates that the graphene behaves similar to a partial polarizer at high frequencies. So the characteristics of the property presented is suggested to be measured via optical absorption experiments.

This work is supported in part by the Australian Research Council, NNSF-China under Grant No. 10674004, and NBRP-China under Grant No. 2006CB921803.

\begin{abstract}
*czhang@uow.edu.au
†mazs@phy.pku.edu.cn

${ }^{1}$ K. S. Novoselov, A. K. Geim, S. V. Morozov, D. Jiang, Y. Zhang, S. V. Dubonos, I. V. Grigorieva, and A. A. Firsov, Science 306, 666 (2004); K. S. Novoselov, D. Jiang, T. Booth, V. V. Khotkevich, S. M. Morozov, and A. K. Geim, Proc. Natl. Acad. Sci. U.S.A. 102, 10451 (2005); K. S. Novoselov, E. McCann, S. V. Morozov, V. I. Falüo, M. I. Katsnelson, U. Zeitler, D. Jiang, F. Schedin, and A. K. Geim, Nat. Phys. 22, 177 (2006).
\end{abstract}

${ }^{2}$ K. S. Novoselov, A. K. Geim, S. V. Morozov, D. Jiang, M. I. Katsenelson, I. V. Grigorieva, S. V. Dubonos, and A. A. Firsov, Nature (London) 438, 197 (2005).

${ }^{3}$ Y. Zhang, Y. W. Tan, H. L. Stormer, and P. Kim, Nature (London) 438, 201 (2005).

${ }^{4}$ H. Suzuura and T. Ando, Phys. Rev. Lett. 89, 266603 (2002); J. Phys. Soc. Jpn. 72, 69 (2003).

${ }^{5}$ S. V. Morozov, K. S. Novoselov, M. I. Katsnelson, F. Schedin, L. A. Ponomarenko, D. Jiang, and A. K. Geim, Phys. Rev. Lett. 97, 016801 (2006).

${ }^{6}$ D. V. Khveshchenko, Phys. Rev. Lett. 97, 036802 (2006).

${ }^{7}$ E. McCann, K. Kechedzhi, V. I. Fal'ko, H. Suzuura, T. Ando, and B. L. Altshuler, Phys. Rev. Lett. 97, 146805 (2006).

${ }^{8}$ Y. S. Zheng and T. Ando, Phys. Rev. B 65, 245420 (2002).

${ }^{9}$ N. M. R. Peres, F. Guinea, and A. H. Castro Neto, Phys. Rev. B 73, 125411 (2006); K. Ziegler, Phys. Rev. Lett. 97, 266802 (2006); I. L. Aleiner and K. B. Efetov, ibid. 97, 236801 (2006); A. Altland, ibid. 97, 236802 (2006); J. Cserti, Phys. Rev. B 75, 033405 (2007); P. M. Ostrovsky, I. V. Gornyi, and A. D. Mirlin, ibid. 74, 235443 (2006); K. Nomura and A. H. MacDonald, Phys. Rev. Lett. 98, 076602 (2007).

${ }^{10}$ M. Fujita, K. Wakabayashi, K. Nakada, and K. K. usakabe, J. Phys. Soc. Jpn. 65, 1920 (1996); Y. Son, M. L. Cohen, and S. G. Louie, Nature (London) 444, 347 (2006); L. Pisani, J. A. Chan,
B. Montanari, and N. Harrison, Phys. Rev. B 75, 064418 (2007); O. V. Yazyev and L. Helm, ibid. 75, 125408 (2007).

${ }^{11}$ M. A. H. Vozmediano, M. P. Lopez-Sancho, T. Stauber, and F. Guinea, Phys. Rev. B 72, 155121 (2005); J. Liu, Z. Ma, A. R. Wright, and C. Zhang, J. Appl. Phys. 103, 103711 (2008).

${ }^{12}$ E. H. Hwang, S. Adam, and S. Das Sarma, Phys. Rev. Lett. 98, 186806 (2007); E. H. Hwang and S. Das Sarma, Phys. Rev. B 75, 205418 (2007).

${ }^{13}$ T. Ando, Y. Zheng, and H. Suzuura, J. Phys. Soc. Jpn. 71, 1318 (2002).

${ }^{14}$ V. P. Gusynin, S. G. Shrapov, and J. P. Carbotte, Phys. Rev. Lett. 96, 256802 (2006).

${ }^{15}$ A. B. Kuzmenko, E. van Heumen, F. Carbone, and D. van der Marel, Phys. Rev. Lett. 100, 117401 (2008).

${ }^{16}$ R. R. Nair, P. Blake, A. N. Grigorenko, K. S. Novoselov, T. J. Booth, T. Stauber, N. M. R. Peres, and A. K. Geim, Science 320, 1308 (2008).

${ }^{17}$ E. G. Mishchenko and B. I. Halperin, Phys. Rev. B 68, 045317 (2003).

${ }^{18}$ R. Saito, G. G. Samsonidze, G. Dresselhaus, M. S. Dresselhaus, A. Jorio, L. G. Cancado, M. A. Pimenta, and A. G. Souza Filho, Appl. Phys. A: Mater. Sci. Process. 78, 1099 (2004); A. Grüneis, R. Saito, G. G. Samsonidze, T. Kimura, M. A. Pimenta, A. Jorio, A. G. Souza Filho, G. Dresselhaus, and M. S. Dresselhaus, Phys. Rev. B 67, 165402 (2003).

${ }^{19}$ Y. Oyama, R. Saito, J. Jiang, G. G. Samsonidze, A. Grüneis, Y. Miyauchi, S. Maruyama, A. Jorio, G. Dresselhaus, and M. S. Dresselhaus, Carbon 44, 873 (2006).

${ }^{20}$ D. S. Abergel, A. Russell, and Vladimir I. Falko, Appl. Phys. Lett. 91, 063125 (2007).

${ }^{21}$ P. Blake, E. W. Hill, A. H. Castro Neto, K. S. Novoselov, D. Jiang, R. Yang, T. J. Booth, and A. K. Geim, Appl. Phys. Lett. 91, 063124 (2007). 